\title{
One-pot four component synthesis of novel 3-furyl coumarin derivatives
}

\author{
VENKATA PRASAD JALLI*, SUVRATHA KRISHNAMURTHY, TETSUJI MORIGUCHI and \\ AKIHIKO TSUGE \\ Department of Applied chemistry, Kyushu Institute of Technology, Kitakyushu, 8048550, Fukuoka, Japan \\ e-mail: jvprasad.008@gmail.com
}

MS received 16 September 2015; revised 21 October 2015; accepted 20 November 2015

\begin{abstract}
Efficient and facile synthesis of 3-furyl coumarin derivatives have been achieved by reaction of 4-chloro-3-formylcoumarin, secondary amines, dialkyl acetylenedicarboxylates and diversely substituted isocyanides using four component, one-pot reaction. All the products were isolated as yellow color fluorescent solids by column chromatography in quantitative yield and characterized with ${ }^{1} \mathrm{H} N M R,{ }^{13} \mathrm{C} N M R$, IR and FAB mass.
\end{abstract}

Keywords. 4-chloro-3-formyl coumarin; secondary amines; [3+2] cyclo addition; Multicomponent reaction; X-ray crystallography.

\section{Introduction}

Coumarin analogs have been considered as an important class of heterocyclic compounds due to their significant applications as anticoagulant, ${ }^{1}$ antibacterial, ${ }^{2}$ antihypertensive, ${ }^{3}$ anti-tubercular, ${ }^{4}$ antifungal,${ }^{5}$ anticancer, ${ }^{6}$ HIV protease inhibition, ${ }^{7}$ Laser dyes and fluorescent dyes. ${ }^{8}$ Similarly, furan ring is found in many pharmaceutically important substances like furanose form in carbohydrate, alkaloid pylocarpine and furacilin antibiotics. Coumarin analogs having both coumarin and furan heterocycles have gained considerable attention of researchers due to their significant properties as anti-leishmania panamensis, ${ }^{9}$ dyes and fluorescent sensors. ${ }^{10}$ Coumarin analogs with furan heterocycle isolated from the plants such as microminutin, micromelin, psoralen, 8-methoxypsoralen have important properties in medicinal chemistry and biophotochemistry. ${ }^{11}$ It is well documented that by introducing a heteroaromatic substituent at 3-position, the absorption and emission maxima of coumarin scaffold can be improved because of extended $\pi$ conjugation and consequently their optoelectronic properties can be improved. ${ }^{12}$ Based on these phenomena a variety of 3-heteroaryl coumarin derivatives have been synthesized and evaluated for their optoelectronic properties. ${ }^{13} 3$-furyl coumarin falls under this class with extended $\pi$ conjugation. Thus the structural features and the wide spectrum of applications of furyl coumarin analogs have prompted the

*For correspondence intense research to develop novel, simple and efficient methods for their synthesis.

There are many reports in literature for the synthesis of 3-furyl coumarin derivatives. These involve the condensation of substituted salicylaldehydes and furyl acetonitriles with subsequent hydrolysis of the resulting 2-imino coumarin intermediates in acidic medium, ${ }^{14}$ NBS bromination of coumarin at 3-position followed by Suzuki-Miyaura cross-coupling reaction with furano boronic acids, ${ }^{15}$ condensation of 4-chloromethyl coumarins with salicylaldehydes in DMF in the presence of potassium carbonate ${ }^{16}$ and condensation of phenol with 2-furyl acid chloride, ${ }^{17}$ followed by Fries rearrangement with benzoyl chloride under PTC conditions and condensation of coumarin chalcone hybrid with benzoyl chloride using TPP catalyst. ${ }^{18}$ Most of the above protocols involved multistep synthesis, metal catalysts, high temperatures, limited substrate scope, tedious workup and long reaction times (scheme 1).

A contemporary challenge in the world is environmental pollution. Multi component reactions are certainly one of the solutions for this problem due to the less release of chemical wastage compared with classical bicomponent reaction protocols and it is being considered as one of the green technology. Previously we have reported the efficient and convenient synthesis of novel 2-benzazepines and chromeno quinolines using green technology platforms. ${ }^{19}$ In this article, we have envisaged a four component one-pot reaction using 4chloro-3-formyl coumarin, secondary amines, dialkyl acetylene dicarboxylate, and isocyanides. The reaction 


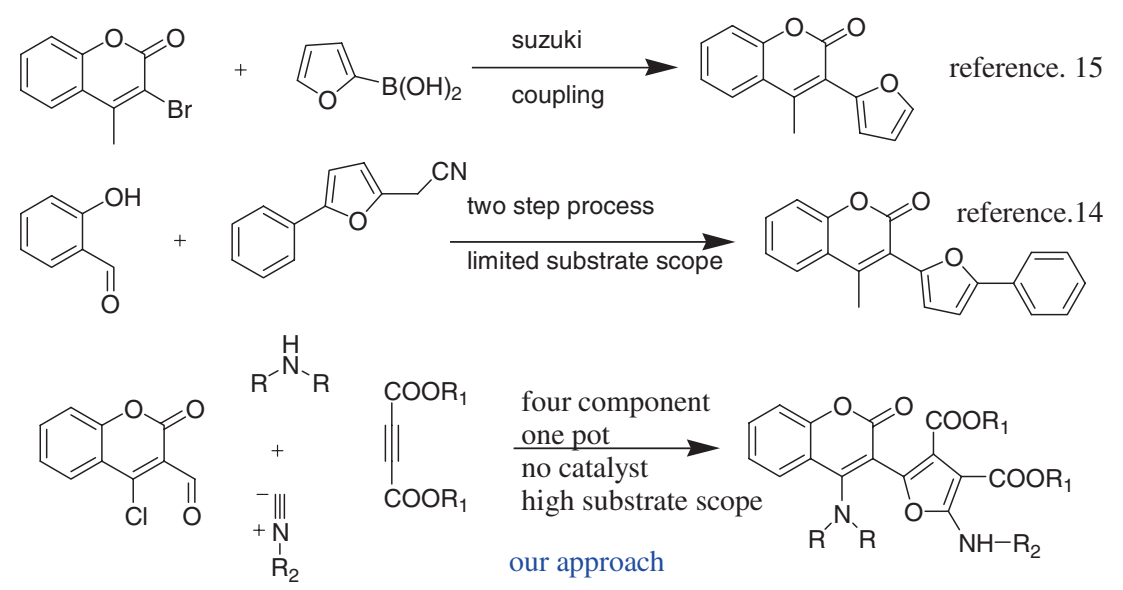

Scheme 1. Comparison of previous reports with our approach.

proceeded smoothly and yielded the desired products in good to excellent yields (83 to $88 \%$ ).

\section{Experimental}

\subsection{General}

All the reagents were purchased from TCI and Sigma Aldrich and used without further purification. All the products were characterized by ${ }^{1} \mathrm{H} \mathrm{NMR},{ }^{13} \mathrm{C} \mathrm{NMR}$, IR, and Fab-Mass analysis. The NMR spectrum was recorded on a Bruker AMX-500 $\mathrm{MHz}$ instrument at room temperature in $\mathrm{CDCl}_{3}$ using TMS as an internal reference. Melting points were determined by AS ONE instrument. Absorption spectrum was measured by using JASCO V-550 UV/VIS Spectrophotometer and Fluorescence spectrum was measured by using Hitachi F-2500 Fluorescence Spectrophotometer. X-ray data for the compound were collected at room temperature using a Bruker Apex II KY CCD diffractometer with graphite monochromated $\mathrm{MoK} \alpha$ radiation $(\lambda=0.71073 \AA)$ with $\omega$-scan method. Crystallographic data of 1 (see section 2.2a) has been deposited with the Cambridge Crystallographic Data Centre as supplementary publication no. CCDC 1424464 contains the supplementary crystallographic data for this paper. These data can be obtained free of charge at www. ccdc.cam.ac.uk/conts/retrieving.html [or from the Cambridge Crystallographic Data Centre (CCDC), 12 Union Road, Cambridge CB2 1EZ, UK; fax: +44(0) 1223336 033; email: deposit@ccdc.cam.ac.uk].

\subsection{General procedure for the synthesis of 3-furyl coumarin derivatives}

A solution of 4-chloro-3-formyl coumarin (1 mmol) and secondary amine $(2 \mathrm{mmol})$ were stirred at reflux for $2 \mathrm{~min}$ in benzene under $\mathrm{N}_{2}$. To this solution dialyl acetylenedicarboxylate $(1 \mathrm{mmol})$, isocyanide $(1 \mathrm{mmol})$ were added and refluxed at $80^{\circ} \mathrm{C}$ for $2.5 \mathrm{~h}$. The volatiles were removed under reduced pressure. The crude reaction mixture was subjected to column chromatography using EtOAc/Hexane mobile phase. All the products were isolated as yellow color fluorescent solids in very good yields.

2.2a 4-morpholino-3-(2-N-t-butylamino-3,4-dimethylcarboxylate-5-furyl) 2H-1-Benzopyran-2-one (1): Yellow color solid, M.p. $133-136^{\circ} \mathrm{C}$; IR: $v_{\max }(\mathrm{KBr})$ 3288, 1732, 1728, 1667, 1658, 1618, 1418, 1240, 1041 $\mathrm{cm}^{-1} ; \delta_{\mathrm{H}}\left(500 \mathrm{MHz} \mathrm{CDCl}_{3}\right) 7.69(1 \mathrm{H}, J=1.2 \mathrm{~Hz}, \mathrm{dd})$ $7.54(\mathrm{~m}, 1 \mathrm{H}), 7.34(1 \mathrm{H}, J=1.0 \mathrm{~Hz}, \mathrm{dd}), 7.29(\mathrm{~m}, 1$ H), $7.02(1 \mathrm{H}, \mathrm{s}), 3.85(4 \mathrm{H}, J=4.55 \mathrm{~Hz}, \mathrm{t}), 3.78(3 \mathrm{H}$, s), $3.75(3 \mathrm{H}, \mathrm{s}), 3.25(4 \mathrm{H}, \mathrm{m}), 1.43(9 \mathrm{H}, \mathrm{s}) ; \delta_{\mathrm{C}}(125$ $\left.\mathrm{MHz}, \mathrm{CDCl}_{3}\right) 165.4,163.9,162.7,161.2,159.5,153.7$, $138.1,132.2,125.3,123.2,118.6,117.8,117.5,102.7$, 87.3, 67.0, 52.7, 52.0, 51.1, 30.1; LCMS: $\mathrm{MH}^{+}, 484$. Anal. Calcd. For $\mathrm{C}_{25} \mathrm{H}_{28} \mathrm{~N}_{2} \mathrm{O}_{8}$ : C, 61.94; H, 5.86; N, 5.78\%. Found: C, 61.95; H, 5.83; N, 5.80\%.

2.2b 4-morpholino-3-(2-N-cyclohexylamino-3,4-dimethylcarboxylate-5-furyl) 2H-1-Benzopyran-2-one (2): Yellow color solid, M.p. $109-112^{\circ} \mathrm{C}$; IR; $v_{\max }(\mathrm{KBr})$ 3288, 1733, 1728, 1667, 1658, 1618, 1418, 1240, 1041 $\mathrm{cm}^{-1} ; \delta_{\mathrm{H}}\left(500 \mathrm{MHz}_{\mathrm{CDCl}}\right) 7.69(1 \mathrm{H}, J=1.4 \mathrm{~Hz}, \mathrm{dd})$, $7.52(1 \mathrm{H}, \mathrm{m}), 7.34(1 \mathrm{H}, J=1.0 \mathrm{~Hz}, \mathrm{dd}), 7.28(1 \mathrm{H}$, $\mathrm{m}), 6.78(1 \mathrm{H}, J=6.8 \mathrm{~Hz}, \mathrm{~d}), 3.88(4 \mathrm{H}, J=4.5 \mathrm{~Hz}$, t), $3.79(3 \mathrm{H}, \mathrm{s}), 3.78(3 \mathrm{H}, \mathrm{s}), 3.19(4 \mathrm{H}, J=4.5 \mathrm{~Hz}, \mathrm{t})$, $2.05(3 \mathrm{H}, \mathrm{m}), 1.76(2 \mathrm{H}, \mathrm{m}), 1.61(1 \mathrm{H}, \mathrm{m}), 1.34(3 \mathrm{H}$, $\mathrm{m}) ; \delta_{\mathrm{C}}\left(125 \mathrm{MHz}, \mathrm{CDCl}_{3}\right)$ 165.2, 164.0, 162.1, 160.9, 159.1, 153.5, 137.6, 132.1, 125.1, 123.8, 119.2, 117.8, 103.7, 86.5, 67.0, 52.1, 51.1, 51.0, 33.7, 33.7, 25.3, 24.6; LCMS: $\mathrm{MH}^{+}$, 510. Anal. Calcd. For $\mathrm{C}_{27} \mathrm{H}_{30} \mathrm{~N}_{2} \mathrm{O}_{8}$ : 
C, 63.50; H, 5.92; N, 5.51\%. Found: C, 63.50; H, 5.90; $\mathrm{N}, 5.53 \%$.

2.2c 4-thiomorpholino-3-(2-N-t-butylamino-3,4-dimethylcarboxylate-5-furyl) 2H-1-Benzopyran-2-one (3): Yellow color solid, M.p. $107-109^{\circ} \mathrm{C}$; IR; $v_{\max }(\mathrm{KBr})$ 3288, 1732, 1728, 1667, 1658, 1618, 1418, 1240, 1041 $\mathrm{cm}^{-1} ; \delta_{\mathrm{H}}\left(500 \mathrm{MHz} \mathrm{CDCl}_{3}\right) 7.69(1 \mathrm{H}, J=1.2 \mathrm{~Hz}, \mathrm{dd})$, $7.52(1 \mathrm{H}, J=8 \mathrm{~Hz}, \mathrm{t}), 7.28-7.33(2 \mathrm{H}, J=1.0 \mathrm{~Hz}$, dd), $7.05(1 \mathrm{H}, \mathrm{s}), 3.78(3 \mathrm{H}, \mathrm{s}), 3.75(3 \mathrm{H}, \mathrm{s}), 3.39-3.48$ $(4 \mathrm{H}, \mathrm{m}), 2.77-2.81(4 \mathrm{H}, \mathrm{m}), 1.44(9 \mathrm{H}, \mathrm{s}) ; \delta_{\mathrm{C}}(125$ $\left.\mathrm{MHz}, \mathrm{CDCl}_{3}\right)$ 165.6, 163.9, 163.0, 161.2, 160.6, 153.4, 138.2, 132.4, 125.1, 123.7, 118.7, 118.1, 117.7, 103.6, 87.8, 53.2, 52.7, 51.8, 51.3, 28.0; LCMS: $\mathrm{MH}^{+}, 500$. Anal. Calcd. For $\mathrm{C}_{25} \mathrm{H}_{28} \mathrm{~N}_{2} \mathrm{O}_{7} \mathrm{~S}: \mathrm{C}, 59.90 ; \mathrm{H}, 5.69 ; \mathrm{N}$, 5.64\%. Found: C, 59.85; H, 5.71; N, 5.67\%.

2.2d 4-thiomorpholino-3-(2-N-cyclohexylamino-3,4dimethylcarboxylate-5-furyl) 2H-1-Benzopyran-2-one (4): Yellow color solid, M.p. $86-88^{\circ} \mathrm{C}$; IR; $v_{\max }(\mathrm{KBr})$ 3288, 1732, 1728, 1667, 1658, 1618, 1418, 1240, 1041 $\mathrm{cm}^{-1} ; \delta_{\mathrm{H}}\left(500 \mathrm{MHz} \mathrm{CDCl}_{3}\right) 7.82(1 \mathrm{H}, J=1.2 \mathrm{~Hz}$, dd), $7.70(1 \mathrm{H}, J=6.8 \mathrm{~Hz}, \mathrm{~d}), 7.32(1 \mathrm{H}, \mathrm{m}), 7.25$ (1 $\mathrm{H}, \mathrm{m}), 6.81(1 \mathrm{H}, J=7.2 \mathrm{~Hz}, \mathrm{~d}), 3.79(3 \mathrm{H}, \mathrm{s}), 3.75$ (3 H, s), 3.38-3.41 (4 H, m), 2.78-2.83 (4 H, m), 1.62 $(1 \mathrm{H}, \mathrm{m}), 1.17-1.38(10 \mathrm{H}, \mathrm{m}) ; \delta_{\mathrm{C}}\left(125 \mathrm{MHz}, \mathrm{CDCl}_{3}\right)$ 162.2, 162.1, 161.4, 160.7, 159.6, 153.4, 146.9, 134.1, $133.7,132.0,126.4,126.1,124.9,119.2,118.6,117.9$, $105.3,87.0,60.9,56.1,52.9,52.0,51.1,33.8,28.7$, 27.9, 25.3, 24.6; LCMS: $\mathrm{MH}^{+}$, 526. Anal. Calcd. For $\mathrm{C}_{27} \mathrm{H}_{30} \mathrm{~N}_{2} \mathrm{O}_{7} \mathrm{~S}: \mathrm{C}, 61.50 ; \mathrm{H}, 5.82 ; \mathrm{N}, 5.32 \%$. Found: $\mathrm{C}$, $61.55 ; \mathrm{H}, 5.75 ; \mathrm{N}, 5.34 \%$.

2.2e 4-piperidino-3-(2-N-t-butylamino-3,4-dimethylcarboxylate-5-furyl) 2H-1-Benzopyran-2-one (5): Yellow color solid, M.p. $92-94^{\circ} \mathrm{C}$; IR; $v_{\max }(\mathrm{KBr}) 3288$, 1732, 1728, 1667, 1658, 1618, 1418, 1240, $1041 \mathrm{~cm}^{-1}$; $\delta_{\mathrm{H}}\left(500 \mathrm{MHz} \mathrm{CDCl}_{3}\right) 7.72(1 \mathrm{H}, J=1.2 \mathrm{~Hz}, \mathrm{dd}), 7.51$ $(1 \mathrm{H}, J=6.9 \mathrm{~Hz}, \mathrm{t}), 7.32(1 \mathrm{H}, \mathrm{m}), 7.26(1 \mathrm{H}, \mathrm{m}), 7.0$ $(1 \mathrm{H}, \mathrm{s}), 3.78(3 \mathrm{H}, \mathrm{s}), 3.73(3 \mathrm{H}, \mathrm{s}), 3.08-3.22(4 \mathrm{H}$, m), $1.73(6 \mathrm{H}, \mathrm{m}), 1.43(9 \mathrm{H}, \mathrm{s}) ; \delta_{\mathrm{C}}\left(125 \mathrm{MHz}, \mathrm{CDCl}_{3}\right)$ $165.6,164.2$, 162.7, 161.6, 160.6, 153.6, 139.2, 131.9, $125.5,123.5,118.2,118.0,118.0,101.4,87.2,60.4$, 52.6, 52.3, 51.9, 51.0, 30.0, 26.3, 24.0, 14.2; LCMS: $\mathrm{MH}^{+}, 482$. Anal. Calcd. For $\mathrm{C}_{26} \mathrm{H}_{30} \mathrm{~N}_{2} \mathrm{O}_{7}$ : C, 64.68; $\mathrm{H}$, 6.29 ; N, 5.83\%. Found: C, 64.70; H, 6.28; N, 5.82\%.

$2.2 \mathrm{f}$ 4-piperidino-3-(2-N-cyclohexylamino-3,4-dimethylcarboxylate-5-furyl) 2H-1-Benzopyran-2-one (6): Yellow color solid, M.p. 93-96 ${ }^{\circ}$; IR; $v_{\max }(\mathrm{KBr}) 3288$, 1732, 1728, 1667, 1658, 1618, 1418, 1240, $1041 \mathrm{~cm}^{-1}$;
$\delta_{\mathrm{H}}\left(500 \mathrm{MHz} \mathrm{CDCl}_{3}\right) 7.71(1 \mathrm{H}, J=1.0 \mathrm{~Hz}, \mathrm{dd}), 7.50(1$ $\mathrm{H}, J=6.5 \mathrm{~Hz}, \mathrm{t}), 7.30(1 \mathrm{H}, \mathrm{m}), 7.26(1 \mathrm{H}, \mathrm{m}), 6.76(1$ $\mathrm{H}, J=7.2 \mathrm{~Hz}, \mathrm{~d}), 3.79(3 \mathrm{H}, \mathrm{s}), 3.75(3 \mathrm{H}, \mathrm{s}), 3.11-3.15$ $(4 \mathrm{H}, \mathrm{m}), 2.01(3 \mathrm{H}, \mathrm{m}), 1.59-1.73(11 \mathrm{H}, \mathrm{m}), 1.33-1.36$ $(3 \mathrm{H}, \mathrm{m}) ; \delta_{\mathrm{C}}\left(125 \mathrm{MHz}, \mathrm{CDCl}_{3}\right) 165.4,164.1,162.1$, $161.3,160.3,153.5,138.8,131.8,125.3,123.6,118.5$, 118.3, 117.6, 102.4, 86.4, 60.4, 52.2, 51.9, 51.4, 51.3, 33.7, 26.2, 25.3, 24.6, 24.1, 14.2; LCMS: $\mathrm{MH}^{+}, 508$. Anal. Calcd. For $\mathrm{C}_{28} \mathrm{H}_{32} \mathrm{~N}_{2} \mathrm{O}_{7}: \mathrm{C}, 66.10 ; \mathrm{H}, 6.35 ; \mathrm{N}$, $5.53 \%$. Found: C, $66.11 ; \mathrm{H}, 6.36 ; \mathrm{N}, 5.51 \%$.

$2.2 \mathrm{~g}$ 4-N-dimethylamino-3-(2-N-t-butylamino-3,4-dimethylcarboxylate-5-furyl) 2H-1-Benzopyran-2-one (7): Yellow color solid, M.p. $66-68^{\circ}$ C; IR; $v_{\max }(\mathrm{KBr}) 3288$, $1732,1728,1667,1658,1618,1418,1240,1041 \mathrm{~cm}^{-1}$; $\delta_{\mathrm{H}}\left(500 \mathrm{MHz} \mathrm{CDCl}_{3}\right) 7.88(1 \mathrm{H}, J=1.2 \mathrm{~Hz}, \mathrm{dd}), 7.51$ $(1 \mathrm{H}, J=6.4 \mathrm{~Hz}, \mathrm{t}), 7.31(1 \mathrm{H}, \mathrm{m}), 7.24(1 \mathrm{H}, \mathrm{m})$, $6.97(1 \mathrm{H}, \mathrm{s}), 3.77(3 \mathrm{H}, \mathrm{s}), 3.74(3 \mathrm{H}, \mathrm{s}), 2.98(6 \mathrm{H}$, s), $1.42(9 \mathrm{H}, \mathrm{s}) ; \delta_{\mathrm{C}}\left(125 \mathrm{MHz}, \mathrm{CDCl}_{3}\right) 165.7,164.1$, $162.7,161.3,160.4,153.6,139.0,132.0,126.4,123.5$, 117.8, 99.9, 86.9, 60.4, 52.6, 52.2, 51.1, 43.4, 29.8; LCMS: $\mathrm{MH}^{+}$, 441. Anal. Calcd. For $\mathrm{C}_{23} \mathrm{H}_{26} \mathrm{~N}_{2} \mathrm{O}_{7}$ : C, 62.43; H, 5.90; N, 6.35\%. Found: C, 62.40; H, 5.95; $\mathrm{N}, 6.33 \%$.

2.2h 4-morpholino-3-(2-N-t-butylamino-3,4-diethylcarboxylate-5-furyl) 2H-1-Benzopyran-2-one (8): Yellow color solid, M.p. $102-105^{\circ} \mathrm{C}$; IR; $v_{\max }(\mathrm{KBr})$ 3288, 1732, 1728, 1667, 1658, 1618, 1418, 1240, 1041 $\mathrm{cm}^{-1} ; \delta_{\mathrm{H}}\left(500 \mathrm{MHz} \mathrm{CDCl}_{3}\right) 7.69(1 \mathrm{H}, J=1.0 \mathrm{~Hz}, \mathrm{dd})$, $7.54(1 \mathrm{H}, \mathrm{m}), 7.34(1 \mathrm{H}, J=1.2 \mathrm{~Hz}, \mathrm{dd}), 7.28(1 \mathrm{H}$, m), $7.04(1 \mathrm{H}, \mathrm{s}), 4.24(4 \mathrm{H}, \mathrm{m}), 3.86(4 \mathrm{H}, J=4.5 \mathrm{~Hz}$, q), 3.21-3.36 (4 H, m), $1.43(9 \mathrm{H}, \mathrm{s}), 1.32(3 \mathrm{H}, J=6.5$ $\mathrm{Hz}, \mathrm{t}), 1.26(3 \mathrm{H}, J=5.7 \mathrm{~Hz}, \mathrm{t}) ; \delta_{\mathrm{C}}\left(125 \mathrm{MHz}, \mathrm{CDCl}_{3}\right)$ $165.1,164.0,162.7,161.2,160.6,153.5,137.8,132.1$, $125.1,123.8,119.0,118.0,117.8,104.0,87.5,60.7$, 59.7, 53.1, 52.6, 30.5, 27.9, 14.1, 13.9; LCMS: $\mathrm{MH}^{+}$, 512. Anal. Calcd. For $\mathrm{C}_{27} \mathrm{H}_{32} \mathrm{~N}_{2} \mathrm{O}_{8}$ : C, 63.25; H, 6.29; N, 5.49\%. Found: C, 63.29; H, 6.27; N, 5.47\%.

$2.2 \mathrm{i}$ 4-N-dimethylamino-3-(2-N-t-butylamino-3,4-diethylcarboxylate-5-furyl) 2H-1-Benzopyran-2-one (9): Yellow color solid, M.p. $158-160^{\circ} \mathrm{C}$; IR; $v_{\max }(\mathrm{KBr})$ 3288, 1735, 1728, 1667, 1658, 1618, 1418, 1240, 1041 $\mathrm{cm}^{-1} ; \delta_{\mathrm{H}}\left(500 \mathrm{MHz} \mathrm{CDCl}_{3}\right) 7.77(1 \mathrm{H}, J=1.2 \mathrm{~Hz}, \mathrm{dd})$, $7.50(1 \mathrm{H}, J=6.5 \mathrm{~Hz}, \mathrm{t}), 7.32(1 \mathrm{H}, \mathrm{m}), 7.25(1 \mathrm{H}, \mathrm{m})$, $6.98(1 \mathrm{H}, \mathrm{s}), 4.24(4 \mathrm{H}, J=4.7 \mathrm{~Hz}, \mathrm{q}), 2.98(6 \mathrm{H}, \mathrm{s})$, $1.42(9 \mathrm{H}, \mathrm{s}), 1.28-1.31(3 \mathrm{H}, J=6.2 \mathrm{~Hz}, \mathrm{t}), 1.22-1.24$ $(3 \mathrm{H}, \mathrm{s}) ; \delta_{\mathrm{C}}\left(125 \mathrm{MHz}, \mathrm{CDCl}_{3}\right) 165.4,164.1,162.8$, $161.6,159.9,153.8,138.5,131.9,126.8,123.4,118.4$, $117.8,99.4,87.4,60.9,59.4,52.3,43.4,29.4,14.4$, 
14.3; LCMS: $\mathrm{MH}^{+}$, 469. Anal. Calcd. For $\mathrm{C}_{25} \mathrm{H}_{30} \mathrm{~N}_{2} \mathrm{O}_{7}$ : C, 63.78; H, 6.45; N, 5.97\%. Found: C, 63.82; H, 6.40; $\mathrm{N}, 5.98 \%$.

$2.2 \mathrm{j}$ 4-thiomorpholino-3-(2-N-cyclohexylamino-3,4diethylcarboxylate-5-furyl) 2H-1-Benzopyran-2-one (10): Yellow color solid, M.p. 89-91 ${ }^{\circ} \mathrm{C}$; IR; $v_{\max }(\mathrm{KBr})$ 3288, 1734, 1728, 1667, 1658, 1618, 1418, 1240, 1041 $\mathrm{cm}^{-1} ; \delta_{\mathrm{H}}\left(500 \mathrm{MHz} \mathrm{CDCl}_{3}\right) 7.69(1 \mathrm{H}, J=1.2 \mathrm{~Hz}, \mathrm{dd})$, $7.52(1 \mathrm{H}, J=6.4 \mathrm{~Hz}, \mathrm{t}), 7.30(1 \mathrm{H}, \mathrm{m}), 7.28(1 \mathrm{H}, \mathrm{m})$, $6.80(1 \mathrm{H}, J=7.2 \mathrm{~Hz}, \mathrm{~d}), 4.24-4.26(4 \mathrm{H}, J=4.4 \mathrm{~Hz}$, q), 3.37-3.40 (4 H, m), 2.78-2.82 (4 H, m), 1.74-1.78 $(3 \mathrm{H}, \mathrm{m}), 1.59-1.61(1 \mathrm{H}, \mathrm{m}), 1.25-1.36(13 \mathrm{H}, \mathrm{m}) ; \delta_{\mathrm{C}}$ $\left(125 \mathrm{MHz}, \mathrm{CDCl}_{3}\right)$ 165.1, 163.7, 162.0, 160.9, 159.9, 153.0, 137.0, 131.7, 124.9, 124.1, 119.8, 118.8, 117.7, $105.4,86.6,60.6,59.5,52.7,51.9,34.7,28.3,24.7$, 24.3, 14.6, 13.9; LCMS: $\mathrm{MH}^{+}$, 554. Anal. Calcd. For $\mathrm{C}_{29} \mathrm{H}_{34} \mathrm{~N}_{2} \mathrm{O}_{7} \mathrm{~S}$ : C, 62.80; H, 6.15; N, 5.08\%. Found: C, $62.78 ; \mathrm{H}, 6.19 ; \mathrm{N}, 5.06 \%$.

2.2k 4-piperidino-3-(2-N-t-butylamino-3,4-diethylcarboxylate-5-furyl) 2H-1-Benzopyran-2-one (11): Yellow color solid, M.p. $71-73^{\circ} \mathrm{C}$; IR; $v_{\max }(\mathrm{KBr}) 3288$, 1734, 1728, 1667, 1658, 1618, 1418, 1240, $1041 \mathrm{~cm}^{-1}$; $\delta_{\mathrm{H}}\left(500 \mathrm{MHz} \mathrm{CDCl}_{3}\right) 7.71(1 \mathrm{H}, J=1.0 \mathrm{~Hz}, \mathrm{dd}), 7.52$ $(1 \mathrm{H}, J=6.6 \mathrm{~Hz}, \mathrm{t}), 7.31(1 \mathrm{H}, \mathrm{m}), 7.30(1 \mathrm{H}, \mathrm{m}), 7.01$ $(1 \mathrm{H}, \mathrm{s}), 4.18-4.23(4 \mathrm{H}, J=4.4 \mathrm{~Hz}, \mathrm{q}), 3.08-3.29$ (4 H, m), 1.64-1.72 (6 H, m), $1.43(9 \mathrm{H}, \mathrm{s}), 1.32(3$ $\mathrm{H}, J=6.2 \mathrm{~Hz}, \mathrm{t}), 1.24(3 \mathrm{H}, J=5.7 \mathrm{~Hz}, \mathrm{t}) ; \delta_{\mathrm{C}}(125$ $\left.\mathrm{MHz}, \mathrm{CDCl}_{3}\right)$ 165.2, 164.0, 162.7, 161.6, 160.6, 153.6, $138.7,131.8,125.5,123.5,118.4,118.1,101.6,87.3$, $60.8,59.3,52.5,52.2,30.0,26.3,24.0,14.2,14.1$; LCMS: $\mathrm{MH}^{+}$, 510. Anal. Calcd. For $\mathrm{C}_{28} \mathrm{H}_{34} \mathrm{~N}_{2} \mathrm{O}_{7}$ : C, 65.83; H, 6.75; N, 5.49\%. Found: C, 65.84; H, 6.70; $\mathrm{N}, 5.49 \%$.

2.21 4-piperidino-3-(2-N-cyclohexylamino-3,4-diethylcarboxylate-5-furyl) 2H-1-Benzopyran-2-one (12): Yellow color solid, M.p. $78-80^{\circ}$ C; IR; $v_{\max }(\mathrm{KBr}) 3288$, $1735,1728,1667,1658,1618,1418,1240,1041 \mathrm{~cm}^{-1}$; $\delta_{\mathrm{H}}\left(500 \mathrm{MHz} \mathrm{CDCl}_{3}\right) 7.71(1 \mathrm{H}, J=1.2 \mathrm{~Hz}, \mathrm{dd}), 7.51(1$ $\mathrm{H}, J=6.6 \mathrm{~Hz}, \mathrm{t}), 7.31(1 \mathrm{H}, \mathrm{m}), 7.26(1 \mathrm{H}, \mathrm{m}), 6.77(1$ $\mathrm{H}, J=7.2 \mathrm{~Hz}, \mathrm{~d}), 4.20-4.26(4 \mathrm{H}, \mathrm{m}), 3.14(4 \mathrm{H}, J=6.2$ $\mathrm{Hz}, \mathrm{t}), 1.58-1.74(11 \mathrm{H}, \mathrm{m}), 1.23-1.37(12 \mathrm{H}, \mathrm{m}), 1.24$ $(3 \mathrm{H}, J=5.7 \mathrm{~Hz}, \mathrm{t}) ; \delta_{\mathrm{C}}\left(125 \mathrm{MHz}, \mathrm{CDCl}_{3}\right) 165.0,163.9$, $162.1,161.4,160.4,153.4,138.2,131.8,125.3,123.6$, $118.9,118.3,117.5,102.4,86.6,60.8,59.6,52.3,52.2$, 51.3, 33.7, 26.4, 25.3, 24.5, 24.1, 14.2, 14.1; LCMS: $\mathrm{MH}^{+}$, 536. Anal. Calcd. For $\mathrm{C}_{30} \mathrm{H}_{36} \mathrm{~N}_{2} \mathrm{O}_{7}$ : C, 67.10; $\mathrm{H}$, $6.76 ; \mathrm{N}, 5.27 \%$. Found: C, 67.13; H, 6.78; N, 5.22\%.
$2.2 \mathrm{~m}$ 4-morpholino-3-[2-N-pentyl-N-(4-pentylimino1,2-diethyl-3-ketenimine dicarboxylate) -5-furyl] 2 H-1-Benzopyran-2-one (13): Yellow color semi solid; IR; $v_{\max }(\mathrm{KBr}) 2079,1733,1728,1667,1658,1618$, $1418,1240,1041 \mathrm{~cm}^{-1} ; \delta_{\mathrm{H}}\left(500 \mathrm{MHz} \mathrm{CDCl}_{3}\right) 7.69(1$ $\mathrm{H}, J=1.2 \mathrm{~Hz}, \mathrm{dd}), 7.52(1 \mathrm{H}, \mathrm{m}), 7.33(1 \mathrm{H}, J=1.0$ $\mathrm{Hz}, \mathrm{dd}), 7.28(1 \mathrm{H}, \mathrm{m}), 6.73(1 \mathrm{H}, \mathrm{s}), 4.11-4.29(8 \mathrm{H}$, m), $3.86(4 \mathrm{H}, J=6.7 \mathrm{~Hz}, \mathrm{t}), 3.06-3.19(4 \mathrm{H}, \mathrm{bm})$, 1.52-1.65 (6 H, m), 1.17-1.37 (22 H, m), $0.88(6 \mathrm{H}, \mathrm{m})$; $\delta_{\mathrm{C}}\left(125 \mathrm{MHz}, \mathrm{CDCl}_{3}\right) 162,15,162.11,161.69,161.38$, $160.70,160.18,153.86,132.40,131.15,125.60$, $125.33,123.83,123.72,117.83,117.76,117.16,66.94$, 62.22, 61.17, 60.91, 53.64, 51.68, 50.67, 49.83, 31.06, 29.73, 29.00, 27.80, 22.58, 14.13, 14.04, 13.96, 13.91; LCMS: $\mathrm{MH}^{+}$, 793. Anal. Calcd. For $\mathrm{C}_{42} \mathrm{H}_{55} \mathrm{~N}_{3} \mathrm{O}_{12}$ : C, 63.50; H, 6.98; N, 5.31\%. Found: C, 63.54; H, 6.94; N, $5.33 \%$.

$2.2 \mathrm{n}$ 4-thiomorpholino-3-[2-N-pentyl-N-(4-pentylimino-1,2-diethyl-3-ketenimine dicarboxylate) -5-furyl]2 H-1-Benzopyran-2-one (14): Yellow color semi solid; IR; $v_{\max }(\mathrm{KBr}) 2079,1733,1728,1667,1658,1618$, $1418,1240,1041 \mathrm{~cm}^{-1} ; \delta_{\mathrm{H}}\left(500 \mathrm{MHz} \mathrm{CDCl}_{3}\right) 7.69(1$ H, m), 7.54 (1 H, m), 7.32 (2 H, m), 6.78 (1 H, s), 4.11$4.29(8 \mathrm{H}, \mathrm{m}), 3.38(4 \mathrm{H}, \mathrm{bm}), 2.80(4 \mathrm{H}, \mathrm{t}), 1.53-1.65$ (6 H, m), 1.17-1.37 (22 H, m), 0.89 (6 H, m); $\delta_{\mathrm{C}}(125$ $\left.\mathrm{MHz}, \mathrm{CDCl}_{3}\right) 163.13,162.15,162.10,161.69,160.70$, $160.18,153.87,132.39,131.20,125.60,123.72$,

Table 1. Screening of solvents for the synthesis of 3-furyl coumarin with 4-chloro-3-formyl coumarin, morpholine, dimethyl acetylenedicarboxylate and t-butyl isocyanide. ${ }^{a}$

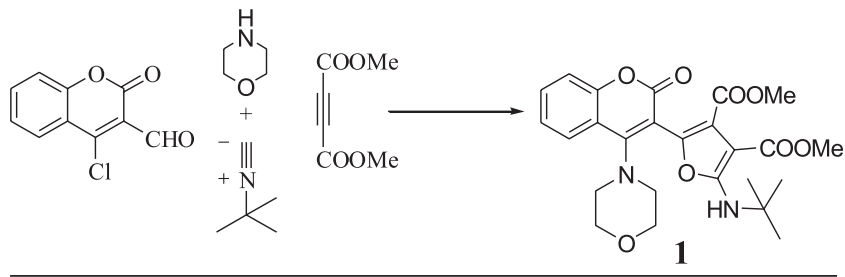

\begin{tabular}{lcccc}
\hline Entry & Solvent & $\mathrm{T}\left({ }^{\circ} \mathrm{C}\right)$ & Time $(\mathrm{h})$ & Yield $^{\mathrm{b}}(\%)$ \\
\hline 1 & $\mathrm{EtOH}$ & 80 & 8 & $\mathrm{NR}^{\mathrm{c}}$ \\
2 & $\mathrm{MeOH}$ & 80 & 8 & $\mathrm{NR}^{\mathrm{c}}$ \\
3 & $\mathrm{DMF}$ & 80 & 8 & $-\mathrm{NR}$ \\
4 & $\mathrm{DCM}$ & 60 & 8 & -40 \\
5 & $\mathrm{CHCl}_{3}$ & 60 & 8 & -40 \\
6 & $\mathrm{THF}$ & 80 & 8 & -50 \\
7 & Toluene & 80 & 3 & -80 \\
8 & Benzene & 80 & 2.5 & -85
\end{tabular}

a Carried out using 4-chloro-3-formyl coumarin (1 mmol), morpholine (2 $\mathrm{mmol})$, dimethyl acetylenedicarboxylate (1 $\mathrm{mmol})$, and t-butyl isocyanide ( $1 \mathrm{mmol}$ ) under $\mathrm{N}_{2}$

$\mathrm{b}$ isolated yields,

c $\mathrm{NR}=$ no reaction. 
Table 2. Synthesis of novel 3-furyl coumarins.

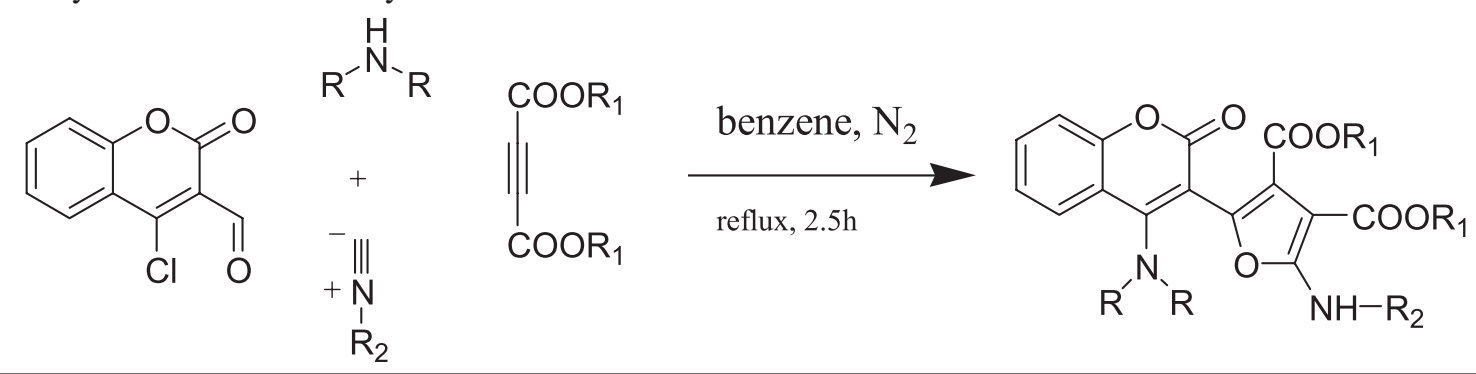

\begin{tabular}{|c|c|c|}
\hline Entry & Secondary amine & Dialkylacetylene dicarboxylate \\
\hline
\end{tabular}

1<smiles>C1COCCN1</smiles><smiles>C1COCCN1</smiles>

2

3

4

5<smiles>C1CSCCN1</smiles><smiles>C1CSCCN1</smiles><smiles>C1CCNCC1</smiles>

6

7

8<smiles>C1CCNCC1</smiles><smiles>CNC</smiles><smiles>C1COCCN1</smiles>

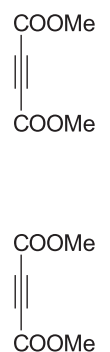

$\left.\right|_{\text {CoOMe }} ^{\text {COOMe }}$

coome

$\left.\right|_{\text {coOMe }} ^{\text {coome }}$
COOMe

||$_{\text {coOMe }}^{\text {COOMe }}$

$\|_{\text {COOEt }}^{\text {COOEt }}$
$Y_{\mathrm{N}}^{+} \equiv$

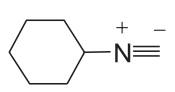

$Y_{\mathrm{N}}^{+} \equiv$

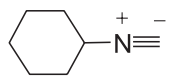

$\mathrm{Y}_{\mathrm{N}}^{+} \equiv$<smiles>CCOCCN1CCCc2ccccc2OCC1</smiles><smiles>COCCN=Cc1ccccc1O</smiles><smiles>COC(=O)c1c(NC(C)C)oc(C2=C(N3CCSCC3)c3ccccc3OC2C)c1C(=O)OC</smiles><smiles></smiles>
4<smiles>COC(=O)c1c(NC(C)C)oc(-c2c(N3CCCCC3)c3ccccc3oc2=O)c1C(=O)OC</smiles>
5<smiles>COC(=O)c1c(NC2CCCCC2)oc(-c2c(N3CCCCC3)c3ccccc3oc2=O)c1C(=O)OC</smiles><smiles>C=NC1CCCCC1</smiles>

$\mathrm{Y}_{\mathrm{N}}^{+} \equiv$

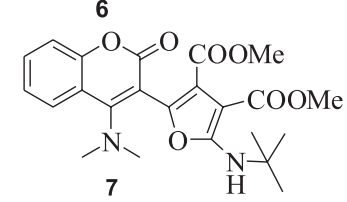<smiles>CCOC(=O)c1c(NC(C)C)oc(-c2c(N3CCOCC3)c(=O)oc3ccccc23)c1C(=O)OCC</smiles>

$Y_{\mathrm{N}}^{+}$ 
Table 2. (continued)

\begin{tabular}{|c|c|c|c|c|c|}
\hline Entry & Secondary amine & Dialkylacetylene dicarboxylate & Isocyanide & 3-Furyl coumarin (1-14) & Yield $(\%)^{\mathrm{a}}$ \\
\hline & & COOEt & & & \\
\hline 9 & -1 & COOEt & & & 87 \\
\hline 10 & & COOEt & & 10 & 83 \\
\hline 11 & & COOEt & & & 85 \\
\hline 12 & & COOEt & & & 85 \\
\hline 13 & & COOEt & $-\mathrm{N}^{+-}$ & & 80 \\
\hline 14 & & COOEt & $-\stackrel{+}{=} \equiv$ & 14 & 80 \\
\hline
\end{tabular}

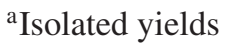

119.03, 117.76, 117.16, 66.94, 62.22, 61.27, 61.17, LCMS: $\mathrm{MH}^{+}$, 809. Anal. Calcd. For $\mathrm{C}_{42} \mathrm{H}_{55} \mathrm{~N}_{3} \mathrm{O}_{11} \mathrm{~S}: \mathrm{C}$, 60.90, 53.64, 51.68, 50.66, 49.83, 31.05, 29.72, 28.98, 62.30; H, 6.80; N, 5.20\%. Found: C, 62.27; H, 6.86; N, $27.80,27.45,22.58,22.48,14.13,14.06,14.04,13.95 ; \quad 5.18 \%$.

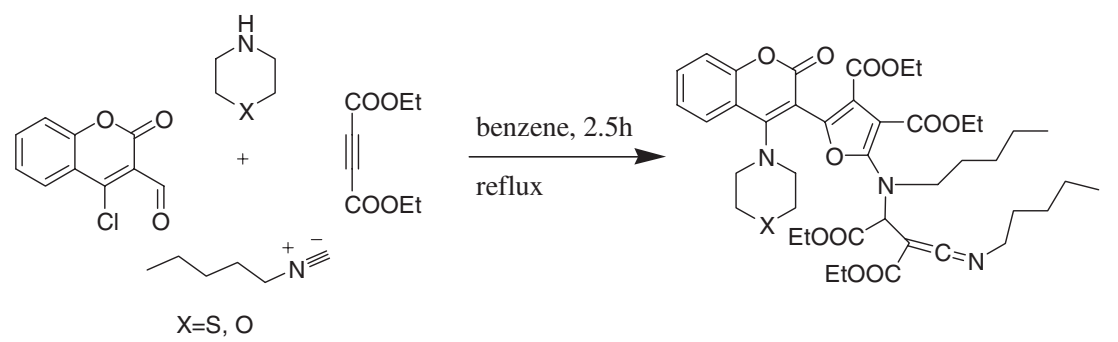

Scheme 2. ${ }^{\text {a }}$ Representation of the formation of products $13,14 .{ }^{\text {a }}$ Reaction was performed using pentyl isocyanide $(2 \mathrm{mmol})$, diethylacetylene dicarboxylate $(2 \mathrm{mmol})$, morpholine (or) thiomorpholine $(2 \mathrm{mmol})$ and 4-chloro-3-formyl coumarin (1 mmol). 


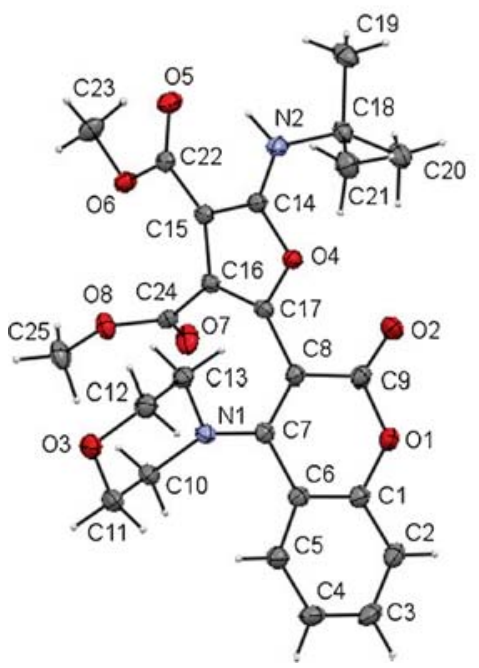

Figure 1. ORTEP plot for the X-ray crystal structure of $\mathbf{1}$ at $50 \%$ probability

\section{Results and Discussion}

Initial attempts by us to successfully synthesize 3 -furyl coumarin derivatives by three component reaction using 4-chloro-3-formyl coumarin, dimethyl acetylenedicarboxylate and tert-butyl isocyanide met with failure. Even when the reaction was conducted at reflux condition it did not yield the desired compounds. Previous reports suggest that 1,3-dipolar generated by the addition of electron deficient alkynes and isocyanides reacts with carbonyl functional group, resulting in furan derivatives. ${ }^{20}$ But, unfortunately in our case, the reaction proceeded to generate a lot of unwanted products as observed by TLC, without the desired product. We envisaged that the problem could be because of chloride of 4-chloro-3-formyl coumarin, as the chloride is a labile group in 4-chloro-3-formyl coumarin molecule, which was known to undergo substitution reactions readily with nucleophiles, hence resulting in many spots on TLC in our case. To our satisfaction, substitution of chloride with morpholine, followed by three component reaction generated the desired product $(85 \%)$. Finally, a four component reaction using 4-chloro-3-formyl coumarin, morpholine, t-butyl isocyanide and dimethyl acetylenedicarboxylate generated the desired product in good yield. The order of addition of reactants was initially 4-chloro-3-formyl coumarin ( 1 eq) and morpholine (2 eq) taken and stirred at reflux for $2 \mathrm{~min}$ in benzene under $\mathrm{N}_{2}$. Subsequently dimethyl acetylenedicarboxylate and t-butyl isocyanide were added and refluxed for $2.5 \mathrm{~h}$.

To choose the best solvent for conducting the reaction, we have optimized the reaction in different solvents under different conditions. No product formation was observed in polar solvents like $\mathrm{EtOH}, \mathrm{MeOH}$ and $\mathrm{DMF}$ at room temperature as well as under reflux at $80^{\circ} \mathrm{C}$ for $8 \mathrm{~h}$ (table 1 , entries $1-3$ ). Poor to moderate yields were observed in $\mathrm{CHCl}_{3}, \mathrm{DCM}$ and THF (table 1, entries 4-6). Very good yields were observed in benzene and toluene (table 1, entries 7-8). Among all the screened solvents, benzene was found to be the more effective solvent with $85 \%$ yield with short reaction time.

Under the optimized reaction conditions, a variety of secondary amines, dialkyl acetylenedicarboxylates and isocyanides were employed to evaluate the substrate scope of the reaction (table 2). All the reactions

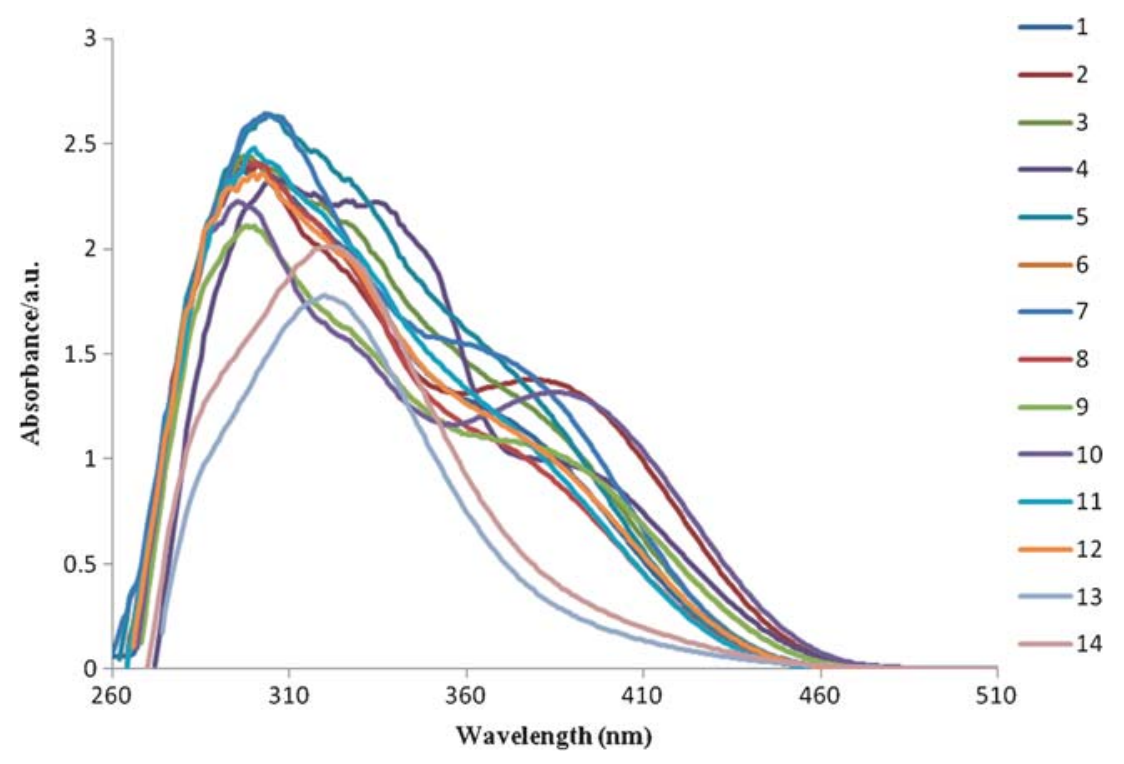

Figure 2. Absorption spectra of compounds 1-14 in $\mathrm{MeOH}$ solvent. 


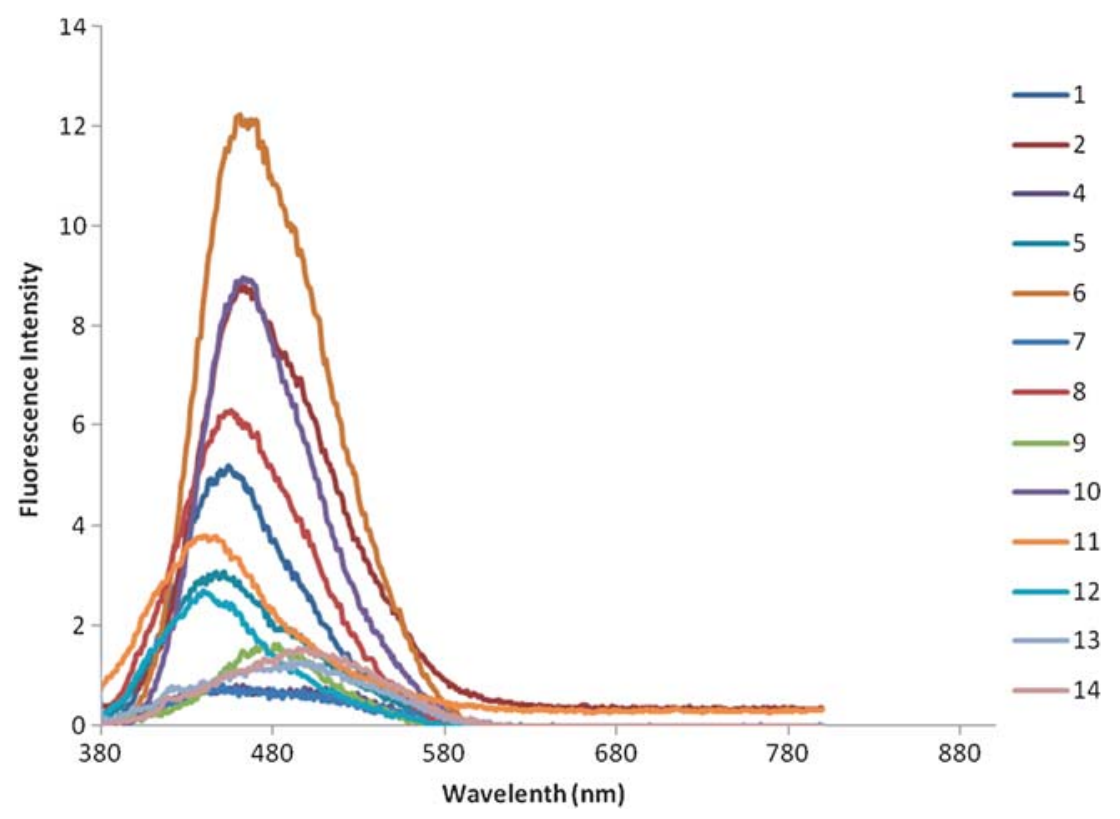

Figure 3. Emission spectra of compounds 1-14 in $\mathrm{MeOH}$.

proceeded very smoothly under optimized reaction conditions. All the 3-furanyl coumarins 1-14 were obtained in good yields. All the compounds reported in this paper are novel, characterized with ${ }^{1} \mathrm{H}$ NMR, ${ }^{13} \mathrm{C}$ NMR, IR and FAB mass.

When the reaction was performed using pentyl isocyanide, diethyl acetylenedicarboxylate, 4-chloro-3formyl coumarin and Morpholine (or) thiomorpholine, we have not observed the usual reaction product in these cases.

When it was further reacted with another molecule of dipolar intermediate formed by the addition of pentyl isocyanide and diethylacetylene dicarboxylate (scheme 3), it formed ketenimine furyl coumarin analogs (products 13, 14), respectively, and the yield of the final product was $40 \%$. When the reaction was conducted by using two equivalents of pentyl isocyanide, diethyl acetylenedicarboxylate as shown (scheme 2), the yield of the final product ketenimine furyl coumarin was increased from 40 to $80 \%$.

The product formations were supported by literature reports, where secondary amines react with dipolar intermediate generated by the addition of electron withdrawing alkynes and isocyanides to form ketenimine products. $^{21}$

We have not observed such kinds of products, when the reaction was attempted using t-butyl isocyanide and cyclohexyl isocyanide. The reason could be because of steric hindrance of bulky t-butyl and cyclohexyl groups which prevented the attack of the second molecule of dipolar intermediate.
Molecule $\mathbf{1}$ was crystallized in ethyl acetate solvent. From the SXRD data, it was concluded that molecule 1 was crystallized in monoclinic form along with ethyl acetate solvent molecule with $\mathrm{p} 21 / \mathrm{c}$ space group. The ORTEP diagram of molecule is shown below (figure 1).

The synthesized compounds (1-14) were further evaluated for their optoelectronic properties. We have measured the UV-Vis absorption and fluorescence emission spectra of compounds $\mathbf{1 - 1 4}$ (figures 2 and 3). The concentrations of 3 -furyl coumarin derivatives

Table 3. UV-Visible absorption and fluorescence data of compounds 1-14

\begin{tabular}{lccc}
\hline Compound & $\lambda_{\text {abs }} / \mathrm{nm}$ & $\lambda_{\mathrm{ex}} / \mathrm{nm}$ & $\lambda_{\mathrm{em}} / \mathrm{nm}$ \\
\hline $\mathbf{1}$ & 299 & 358 & 456 \\
$\mathbf{2}$ & 300 & 355 & 461 \\
$\mathbf{3}$ & 297 & 366 & 440 \\
$\mathbf{4}$ & 308 & 362 & 454 \\
$\mathbf{5}$ & 304 & 355 & 446 \\
$\mathbf{6}$ & 298 & 357 & 465 \\
$\mathbf{7}$ & 303 & 355 & 472 \\
$\mathbf{8}$ & 298 & 357 & 457 \\
$\mathbf{9}$ & 322 & 354 & 476 \\
$\mathbf{1 0}$ & 295 & 351 & 463 \\
$\mathbf{1 1}$ & 298 & 355 & 445 \\
$\mathbf{1 2}$ & 300 & 357 & 444 \\
$\mathbf{1 3}$ & 320 & 372 & 501 \\
$\mathbf{1 4}$ & 322 & 371 & 496 \\
\hline
\end{tabular}

${ }^{a}$ The concentration of all the samples were $2.0 \times 10^{-4} \mathrm{~mol} / \mathrm{L}$ in methanol solvent. 

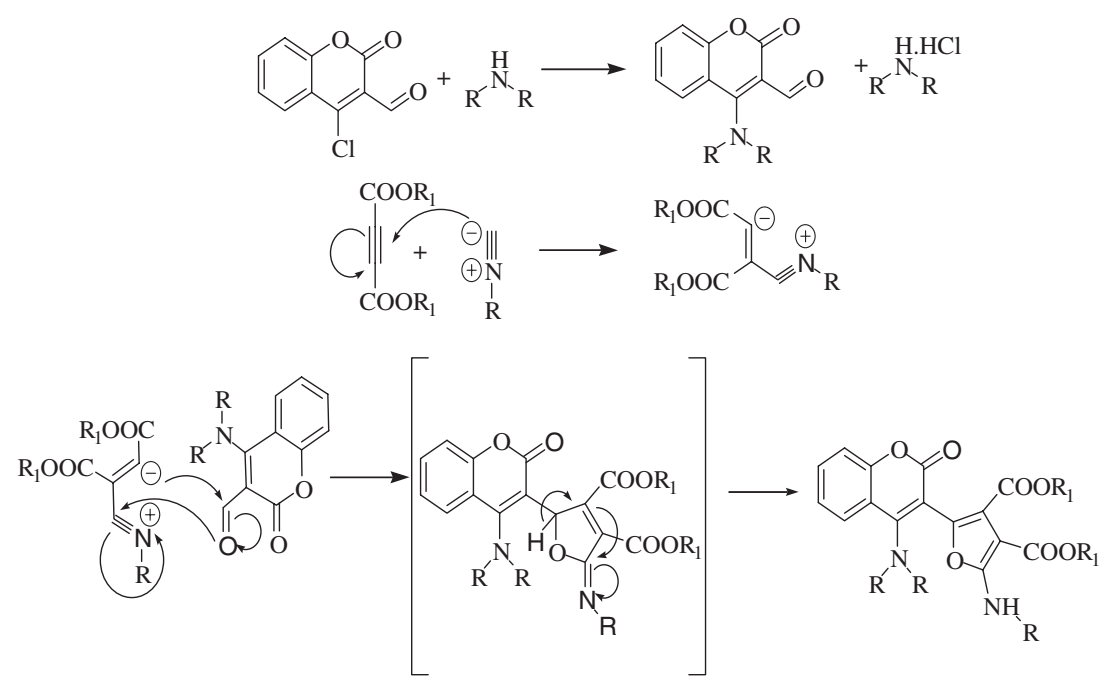

Scheme 3. Plausible reaction mechanism for the formation of 3-furyl coumarin.

were $2.0 \times 10^{-4} \mathrm{~mol} / \mathrm{L}$ for absorption in $\mathrm{MeOH}$ solvent. The results are summarized in table 3. The shapes of absorption spectra of all the compounds are very similar to each other. The absorption spectra (figure 2) have shown absorption maxima from 295 $\mathrm{nm}$ to $322 \mathrm{~nm}$ for all the compounds in methanol solvent.

With the above results in hand, we would like to propose the plausible mechanism for the above reaction. The first step of the reaction was nucleophilic displacement of chloride group with secondary amine as shown in scheme 3.

On the basis of well-established chemistry of isocyanides reactivity with dialkyl acetylenedicarboxylate, a zwitter ionic intermediate was expected. This zwitter ionic intermediate was added to carbonyl function of coumarin and formed an intermediate, which finally undergoes rearrangement to form the final product.

\section{Conclusions}

In conclusion, we have reported a mild and efficient synthesis of 3-furyl coumarins via four component, one-pot reaction. The protocol afforded all the products as yellow color solids after column chromatography in good to excellent yields.

\section{Supplementary Information (SI)}

General experimental section, analytical data for the compounds 1-14 and X-ray analysis for the compound 1 can be found in supplementary information, available at www.ias.ac.in/chemsci. Crystallographic data of $\mathbf{1}$ has been deposited with the Cambridge Crystallographic Data Centre as supplementary publication nos. CCDC 1424464 contains the supplementary crystallographic data for this paper.

\section{Acknowledgements}

We are very thankful to Kyushu Institute of Technology for their kind support and encouragement. We also thank Dr. Kenji Yoza (Bruker AXS Japan) for experimental assistance during final stages of the X-ray analysis.

\section{References}

1. Golfakhrabadi F, Abdollahi M, Ardakani M R S, Saeidnia S, Akbarzadeh T, Ahmadabadi A N, Ebrahimi A, Yousefbeyk F, Hassanzadeh A and Khanavi M 2014 Pharm. Biol. 521335

2. (a) Venugopala $\mathrm{K} \mathrm{N}$ and Jayashree B S 2008 Indian J. Pharm. Sci. 70 88; (b) Matsos M J, Vazquez-Rodriguez S, Santana L, Uriarte E, Fuentes-Edfuf C, Santos Y and Munoz-Crego A 2012 Med. Chem. 81140

3. Amin K M, Awadalla F M, Eissa A A M, Abou-Seri S M and Hassan G S 2011 Bioorg. Med. Chem. 196087

4. Arshad A, Osman H, Bagley M C, Lam C K, Mohammad S and Zahariluddin A S M 2011 Eur. J. Med. Chem. 463788

5. Shi Y and Zhou C H 2011 Bioorg. Med. Chem. 21956

6. Paul K, Bindal S and Luxami V 2013 Bioorg. Med. Chem. 233667

7. Olomola T O, Klein R, Mautsa N, Sayed Y and Kaye P T 2013 Bioorg. Med. Chem. 211964

8. (a) Edwards P D, Mauger R C, Cottrell K M, Morris F X, Pine K K, Sylvester M A, Scott C W and Furlong S T 2000 Bioorg. Med. Chem. Lett. 10 2291; (b) Adamczyk M, Cornwell M, Huff J, Rege S and Rao 
T V S 1997 Bioorg. Med. Chem. Lett. 7 1985; (c) Seidel C A M, Schulz A and Sauer M H M 1996 J. Phys. Chem. 100 5541; (d) Adronov A, Gilat S L, Fre-chet J M, Ohta K, Neuwahl F V R and Fleming G R $2000 \mathrm{~J}$. Am. Chem. Soc. 122 1175; (e) Shaughnessy K H, Kim P and Hartwig J F 1999 J. Am. Chem. Soc. 1212123

9. Arango V, Robledo S, Séon-Méniel B, Figadère B, Cardona W, Saez J and Otalvaro F 2010 J. Nat. Prod. 73 1012

10. (a) Zen A A, Aylott J W and Chan W C 2014 Tetrahedron. Lett. 55 5521; (b) Wlser K, Grilj J, Vauthey E, Aylott J W and Chan W C 2009 Chem. Commun. 671; (c) Schiedel M S, Briehn C A and Bauerle P 2001 Angew. Chem. Int. Ed. 40 4677; (d) Gambari R, Lampronti I, Bianchi N, Zuccato C, Viola G, Vedaldi D and Acqua F D 2007 Heterocycl. Chem. 9 265; (e) Kitamura N, Kohtani S and Nakagaki R 2005 J. Photochem. Photobiol. C 6 168; (f) Santana L, Uriarte E, Roleira F, Milhazes N and Borges F 2004 Curr. Med. Chem.11 3239

11. (a) Murakami A, Gao G, Omura M, Yano M, Ito C, Furukawa H, Takahashi D, Koshimizu K and Ohigashi H 2000Bioorg. Med. Chem. 10 59; (b) Luo X, He W, Yin H, Li Q, Huang Y and Zhang S 2012 Molecules 17 6944; (c) Bethea D, Fullmer B, Syed S, Seltzer G, Tiano J, Rischko C, Gillespie L, Brown D and Gasparro F P 1999 J. Dermatol. Sci. 47 1390; (d) Lowes M A, Bowcock A M and Krueger J G 2007 Nature 445 866
12. Sawa M, Hsu T L, Itoh T, Sugiyama M, Hanson S R, Vogt P K and Wong C H 2006 Proc. Nat. Acad. Sci. U.S.A. 10312371

13. (a) Zen A A, Aylott J W and Chan W C 2014 Tetrahedron. Lett. 55 5521; (b) Wlser K, Grilj J, Vauthey E, Aylott J W and Chan W C 2009 Chem. Commun. 671; (c) Schiedel M S, Briehn C A and Bauerle P 2001 Angew. Chem. Int. Ed. 404677

14. Chodankar N K and Seshadri S 1985 Dyes Pigm. 6331

15. Zen A A, Aylott J W and Chan W C 2014 Tetrahedron. Lett. 555521

16. Khan I A and Kulkarni M V 1999 Indian. J. Chem. 38B 491

17. Udaya Kumari T, David Krupadanam G L and Srimannarayana G 2000 Indian. J. Chem. 39B 62

18. Jang Y J, Syu S E, Chen Y J, Yang M C and Lin W 2013 Org. Biomol. Chem. 10843

19. (a) Prasad J V, Prabhakar M, Manjulatha K, Rambabu D, Solomon K A, Krishna G G and Kumar K A 2010 Tetrahedron Lett. 51 3109; (b) Jaggavarapu S R, Kamalakaran A S, Prasad J V, Sravan kumar G, Ganesh M R and Gopikrishna G 2014J. Chem. Sci. 126187

20. (a) Nair V, Vinod A U, Nair J S, Sreekanth A R and Rath N P 2000 Tetrahedron Lett. 41 6675; (b) Nair V and Vinod A U 2000 Chem. Commun. 1019; (c) Ji F, Sun M, Lv M F, Yi W B and Cai C 2013 Synthesis 45375

21. (a) Yavari I, Djahaniani H and Nasiri F 2004 Collect. Czech. Chem. Commun. 69 1499; (b) Bayat M, Imanieh $\mathrm{H}$ and Hossieninejad E 2008 Synth. Commun. 382567 